\title{
Regioselective Construction of Substituted Conjugated Dienes Using an Olefin Cross-Metathesis Protocol
}

\author{
Timothy W. Funk, Jon Efskind, and Robert H. Grubbs \\ The Arnold and Mabel Beckman Laboratory of Chemical Synthesis, \\ Division of Chemistry and Chemical Engineering, California Institute of \\ Technology, Pasadena, CA 91125
}

General Experimental Section. NMR spectra were recorded on an Oxford $300 \mathrm{MHz}$ NMR spectrometer running Varian VNMR software. Chemical shifts are reported in parts per million (ppm) downfield from tetramethylsilane (TMS) with reference to internal solvent. Multiplicities are abbreviated as follows: singlet (s), doublet (d), triplet (t), quartet (q), quintet (quint), multiplet (m), and broad (br). Spectroscopic data is provided for the major olefin isomer. For all vinylboronates reported the ${ }^{13} \mathrm{C}$ peak of the $\alpha$-carbon is not observed due to the large quadrupolar effect of the attached boron nucleus. $\mathrm{E} / \mathrm{Z}$ ratios given for the products indicate the ratios of the $\mathrm{C}=\mathrm{C}$ bond formed in cross-metathesis and were determined from coupling constants of vinylic protons in the ${ }^{1} \mathrm{H}$ NMR spectrum.

Analytical thin-layer chromatography (TLC) was performed using silica gel 60 F254 precoated plates $(0.25 \mathrm{~mm}$ thickness $)$ with a fluorescent indicator. Visualization was performed with standard potassium permanganate stains or UV light. Flash column chromatography was performed using silica gel 60 (230-400 mesh). All glassware was either oven dried or flame dried, and reactions were done under an atmosphere of argon. All commercial chemicals were used as obtained except 1,4-diacetoxy-cis-2-butene, 
which was distilled from $\mathrm{CaH}_{2}$. 3-Methyl-1,3-pentadiene was received (Aldrich) as a 70:30 mixture of $\mathrm{E} / \mathrm{Z}$ isomers, and both isomers underwent cross-metathesis in the examples below (NMR spectral data is given for both isomers whenever it could be determined). Benzene and methylene chloride were dried by passage through solvent columns containing activated alumina. (2Z,4E)-2-Bromo-ethyl-sorbate ${ }^{\mathrm{i}}(\mathbf{9})$ (which was isolated and used as a 9:1 mixture of $(2 Z, 4 E)$-2-bromo-ethyl-sorbate and $(2 E, 4 E)$-2bromo-ethyl-sorbate), 1,1-dibromo-1,3-pentadiene ${ }^{\mathrm{ii}}$ (15), and 2-hexylbuta-1,3-diene ${ }^{\mathrm{iii}}(\mathbf{3 6})$ were prepared according to literature procedures. Non-commercially available conjugated dienes were prepared as follows:
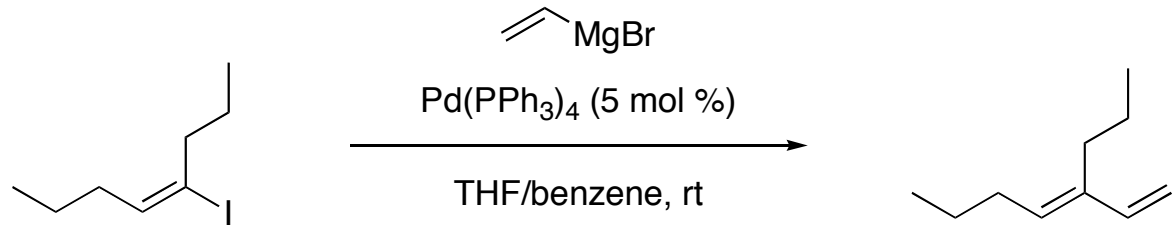

29

Diene 29. To a solution of $\mathrm{Pd}\left(\mathrm{PPh}_{3}\right)_{4}(340 \mathrm{mg}, 0.29 \mathrm{mmol})$ in $30 \mathrm{~mL}$ benzene at $\mathrm{rt}$ was added (E)-4-iodo-4-octene ${ }^{\text {iv }}(1.4 \mathrm{~g}, 5.9 \mathrm{mmol})$ and vinylmagnesium bromide (1M in THF, $11.8 \mathrm{~mL}, 11.8 \mathrm{mmol}$ ). After $3 \mathrm{~h}$, saturated aqueous $\mathrm{NH}_{4} \mathrm{Cl}$ was added and the mixture was extracted with $3 \times 25 \mathrm{~mL} \mathrm{Et}_{2} \mathrm{O}$. The organics were combined, washed with saturated aqueous $\mathrm{NaHCO}_{3}$, water, brine, dried over $\mathrm{MgSO}_{4}$, and concentrated. The crude oil was purified by flash chromatography (hexanes) to give $0.52 \mathrm{~g}(64 \%) 29$ as a colorless liquid. ${ }^{1} \mathrm{H}$ NMR (300 MHz, $\left.\mathrm{CDCl}_{3}, \mathrm{ppm}\right): \delta 6.68(\mathrm{dd}, \mathrm{J}=17.6,11.0 \mathrm{~Hz}, 1 \mathrm{H}), 5.38(\mathrm{t}, \mathrm{J}=7.4 \mathrm{~Hz}$, 1H), $5.21(\mathrm{~d}, \mathrm{~J}=17.9 \mathrm{~Hz}, 1 \mathrm{H}), 5.06(\mathrm{dt}, \mathrm{J}=11.0,1.6 \mathrm{~Hz}, 1 \mathrm{H}), 2.11-2.19(\mathrm{~m}, 4 \mathrm{H}), 1.34-$ $1.57(\mathrm{~m}, 4 \mathrm{H}), 0.91(\mathrm{t}, \mathrm{J}=7.4 \mathrm{~Hz}, 3 \mathrm{H}), 0.90(\mathrm{t}, \mathrm{J}=7.4 \mathrm{~Hz}, 3 \mathrm{H}) .{ }^{13} \mathrm{C}$ NMR $(75 \mathrm{MHz}$, 
$\left.\mathrm{CDCl}_{3}, \mathrm{ppm}\right): \delta 136.6,133.2,130.9,113.0,35.7,29.6,23.3,22.1,14.2,14.0$. HRMS (EI) calc. for $\mathrm{C}_{10} \mathrm{H}_{18}: 138.1409$, found 138.1406 .

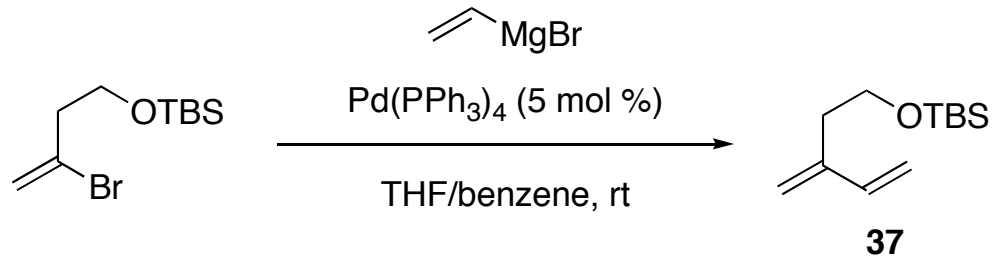

Diene 37. Following the same procedure as 29, 3-bromo-3-buten-1-ol $t$ butyldimethylsilyl ether ${ }^{\mathrm{v}}(1.0 \mathrm{~g}, 3.8 \mathrm{mmol})$, vinylmagnesium bromide (1M in THF, 7.5 $\mathrm{mL}, 7.5 \mathrm{mmol})$, and $\mathrm{Pd}\left(\mathrm{PPh}_{3}\right)_{4}(218 \mathrm{mg}, 0.19 \mathrm{mmol})$ in $20 \mathrm{~mL}$ benzene gave $0.61 \mathrm{~g}$ (76\%) of $\mathbf{3 7}$ as a colorless liquid. ${ }^{1} \mathrm{H} \mathrm{NMR}\left(300 \mathrm{MHz}, \mathrm{CDCl}_{3}, \mathrm{ppm}\right): \delta 6.36$ (dd, $\mathrm{J}=17.6$, $11.3 \mathrm{~Hz}, 1 \mathrm{H}), 5.24(\mathrm{~d}, \mathrm{~J}=17.6 \mathrm{~Hz}, 1 \mathrm{H}), 5.06(\mathrm{~d}, \mathrm{~J}=11.0 \mathrm{~Hz}, 1 \mathrm{H}), 5.06($ broad s, 1H), $5.03($ broad s, 1H), $3.74(\mathrm{t}, \mathrm{J}=7.1 \mathrm{~Hz}, 2 \mathrm{H}), 2.46(\mathrm{t}, \mathrm{J}=7.1 \mathrm{~Hz}, 2 \mathrm{H}), 0.90(\mathrm{~s}, 9 \mathrm{H}) 0.05(\mathrm{~s}$, 6H). $\left.{ }^{13} \mathrm{C} \mathrm{NMR} \mathrm{(75} \mathrm{MHz,} \mathrm{CDCl}_{3}, \mathrm{ppm}\right): \delta$ 143.4, 139.1, 117.6, 113.5, 62.6, 35.2, 26.1, 18.6, -5.0. HRMS (EI) calc. for $\mathrm{C}_{12} \mathrm{H}_{24} \mathrm{OSi}$ : 212.1597, found 212.1592 .

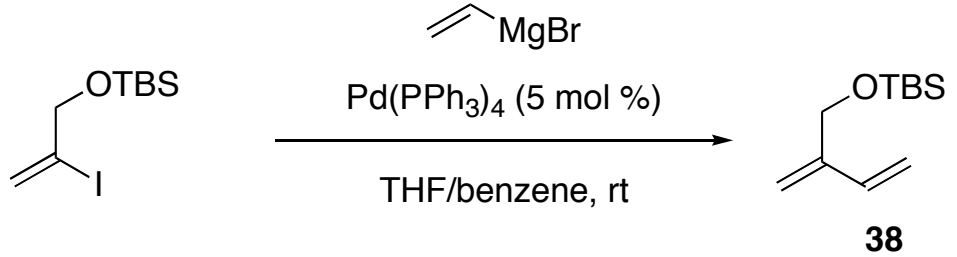


Diene 38. Following the same procedure as 29, 1-(t-butyldimethylsiloxy)-2iodopropene $^{\mathrm{iv}, \mathrm{vi}}(1.3 \mathrm{~g}, 4.4 \mathrm{mmol})$, vinylmagnesium bromide (1M in THF, $8.7 \mathrm{~mL}, 8.7$ $\mathrm{mmol})$, and $\mathrm{Pd}\left(\mathrm{PPh}_{3}\right)_{4}(252 \mathrm{mg}, 0.22 \mathrm{mmol})$ in $23 \mathrm{~mL}$ benzene gave $0.24 \mathrm{~g}(28 \%)$ of 38 as a colorless liquid. ${ }^{1} \mathrm{H} \mathrm{NMR}\left(300 \mathrm{MHz}, \mathrm{CDCl}_{3}, \mathrm{ppm}\right): \delta 6.39(\mathrm{dd}, \mathrm{J}=17.9,11.0 \mathrm{~Hz}$, 1H), 5.33 (br s, 1H), 5.17 (d, J = 18.1 Hz, 1H), 5.11 (br s, 1H), 5.04 (d, J = $11.0 \mathrm{~Hz}, 1 \mathrm{H})$, $4.35(\mathrm{t}, \mathrm{J}=1.5 \mathrm{~Hz}, 2 \mathrm{H}), 0.93(\mathrm{~s}, 9 \mathrm{H}), 0.09(\mathrm{~s}, 6 \mathrm{H}) .{ }^{13} \mathrm{C} \mathrm{NMR}\left(75 \mathrm{MHz}, \mathrm{CDCl}_{3}, \mathrm{ppm}\right): \delta$ 145.1, 136.8, 114.9, 113.2, 62.6, 26.1, 18.6, -5.2. HRMS (EI) calc. for $\mathrm{C}_{11} \mathrm{H}_{22} \mathrm{OSi}$ : 198.1440, found 198.1449.

\section{General Procedure for Cross-Metathesis Reactions with $(2 Z, 4 \mathrm{E})-2-B r o m o-e t h y l-$} sorbate and 1,1-Dibromo-1,3-pentadiene (Table 1). Entry 1, $(2 Z, 4 E)$-ethyl 2bromoundeca-2,4-dienoate (16). To a solution of catalyst 3 (20 mg, $0.023 \mathrm{mmol})$ in 2.3 $\mathrm{mL} \mathrm{CH}_{2} \mathrm{Cl}_{2}$ was added (2Z,4E)-2-bromo-ethyl-sorbate (9) (100 mg, $\left.0.46 \mathrm{mmol}\right)$ and allylbenzene (10) (162 mg, $1.4 \mathrm{mmol})$, and the solution stirred for $12 \mathrm{~h}$ at $40{ }^{\circ} \mathrm{C}$. The volatiles were removed by rotary evaporation, and the residue was purified by flash chromatography to give $92 \mathrm{mg}(68 \%, 8.5: 1 \mathrm{E} / \mathrm{Z}) \mathbf{1 6}$ as a colorless oil. ${ }^{1} \mathrm{H}$ NMR (300 $\left.\mathrm{MHz}, \mathrm{CDCl}_{3}, \mathrm{ppm}\right): \delta 7.70(\mathrm{~d}, \mathrm{~J}=10.5 \mathrm{~Hz}, 1 \mathrm{H}), 7.21-7.39(\mathrm{~m}, 5 \mathrm{H}), 6.56-6.64(\mathrm{~m}, 1 \mathrm{H})$, $6.47(\mathrm{dt}, \mathrm{J}=15.5,6.7 \mathrm{~Hz}, 1 \mathrm{H}), 4.32(\mathrm{q}, \mathrm{J}=7.0 \mathrm{~Hz}, 2 \mathrm{H}), 3.59(\mathrm{~d}, \mathrm{~J}=6.7 \mathrm{~Hz}, 2 \mathrm{H}), 1.37(\mathrm{t}$, $\mathrm{J}=7.0 \mathrm{~Hz}, 3 \mathrm{H}) .{ }^{13} \mathrm{C} \mathrm{NMR}\left(75 \mathrm{MHz}, \mathrm{CDCl}_{3}, \mathrm{ppm}\right): \delta 165.0,145.0,140.92,138.4,128.6$, 128.5, 128.4, 126.5, 113.2, 62.3, 39.7, 14.2. GC-MS (EI): $296\left(\mathrm{M}^{+}\right), 294\left(\mathrm{M}^{+}\right), 215,169$, 141.

Entry 2, (2Z,4E)-ethyl 2-bromo-5-phenylpenta-2,4-dienoate (17). ${ }^{\text {i }}$ Following the general procedure for 16,9 (73 $\mathrm{mg}, 0.33 \mathrm{mmol})$, styrene (11) $(104 \mathrm{mg}, 1.0 \mathrm{mmol})$, and 3 
(30 mg, $0.033 \mathrm{mmol})$ in $1.8 \mathrm{~mL} \mathrm{CH} \mathrm{Cl}_{2}$ gave $60 \mathrm{mg}(65 \%, 10: 1 \mathrm{E} / \mathrm{Z}$ ) of $\mathbf{1 7}$ as a colorless oil. ${ }^{~} \mathrm{H}$ NMR (300 MHz, $\left.\mathrm{CDCl}_{3}, \mathrm{ppm}\right): \delta 7.86(\mathrm{~d}, \mathrm{~J}=10.0 \mathrm{~Hz}, 1 \mathrm{H}), 7.55-7.58(\mathrm{~m}, 2 \mathrm{H})$, 7.38-7.41 (m, 3H), 7.07-7.25 (m, 2H), $4.35(\mathrm{q}, \mathrm{J}=7.0 \mathrm{~Hz}, 2 \mathrm{H}), 1.40(\mathrm{t}, \mathrm{J}=7.0 \mathrm{~Hz}, 3 \mathrm{H})$. ${ }^{13} \mathrm{C}$ NMR (75 MHz, $\left.\mathrm{CDCl}_{3}, \mathrm{ppm}\right): \delta 162.9,142.6,141.1,135.9,129.5,128.8,127.5$, $125.2,114.3,62.4,14.2$.

Entry 3, (2Z,4E)-ethyl 6-acetoxy-2-bromohexa-2,4-dienoate (18). Following the general procedure for $\mathbf{1 6}, \mathbf{9}(132 \mathrm{mg}, 0.60 \mathrm{mmol})$, 1,4-diacetoxy-cis-2-butene (12) (311 $\mathrm{mg}, 1.80 \mathrm{mmol}$ ), and $\mathbf{3}(51 \mathrm{mg}, 0.060 \mathrm{mmol})$ in $3 \mathrm{~mL} \mathrm{CH}_{2} \mathrm{Cl}_{2}$ gave $117 \mathrm{mg}(70 \%, 7.5: 1$ E/Z) of 18 as a colorless oil. ${ }^{1} \mathrm{H}$ NMR (300 MHz, $\left.\mathrm{CDCl}_{3}, \mathrm{ppm}\right): \delta 7.63(\mathrm{~d}, \mathrm{~J}=10.6 \mathrm{~Hz}$, 1H), 6.67 (ddt, $\mathrm{J}=15.5,10.7,1.7 \mathrm{~Hz}, 1 \mathrm{H}), 6.29-6.38(\mathrm{ddt}, \mathrm{J}=15.5,5.6,0.9 \mathrm{~Hz}, 1 \mathrm{H}), 4.73$ $(\mathrm{dd}, \mathrm{J}=5.6,1.5 \mathrm{~Hz}, 2 \mathrm{H}), 4.32(\mathrm{q}, \mathrm{J}=7.0 \mathrm{~Hz}, 2 \mathrm{H}), 2.14(\mathrm{~s}, 3 \mathrm{H}), 1.37(\mathrm{t}, \mathrm{J}=7.0 \mathrm{~Hz}, 3 \mathrm{H})$. ${ }^{13} \mathrm{C}$ NMR (75 MHz, $\left.\mathrm{CDCl}_{3}, \mathrm{ppm}\right): \delta$ 170.5, 163.7, 139.7, 137.9, 129.4, 115.7, 63.8, 62.9, 20.8, 14.1. GC-MS (EI): $278\left(\mathrm{M}^{+}\right), 276\left(\mathrm{M}^{+}\right), 215,169,141$.

Entry 4, (2Z,4E)-ethyl 2-bromo-6-chlorohexa-2,4-dienoate (19). Following the general procedure for 16,9 (92 mg, $0.42 \mathrm{mmol}$ ), 1,4-dichloro-cis-2-butene (13) (105 mg, $0.84 \mathrm{mmol})$, and $\mathbf{3}(18 \mathrm{mg}, 0.021 \mathrm{mmol})$ in $2 \mathrm{~mL} \mathrm{CH}_{2} \mathrm{Cl}_{2}$ gave $51 \mathrm{mg}(48 \%, 6: 1 \mathrm{E} / \mathrm{Z})$ of 19 as a colorless oil. ${ }^{1} \mathrm{H}$ NMR (300 MHz, $\left.\mathrm{CDCl}_{3}, \mathrm{ppm}\right): \delta 7.64(\mathrm{~d}, \mathrm{~J}=10.5 \mathrm{~Hz}, 1 \mathrm{H}), 6.72$ $(\mathrm{dd}, \mathrm{J}=15.5,10.5 \mathrm{~Hz}, 1 \mathrm{H}), 6.56(\mathrm{dt}, \mathrm{J}=15.5,7.2 \mathrm{~Hz}, 1 \mathrm{H}), 4.29(\mathrm{q}, \mathrm{J}=7.2 \mathrm{~Hz}, 2 \mathrm{H}), 4.17$ $(\mathrm{d}, \mathrm{J}=7.2 \mathrm{~Hz}, 2 \mathrm{H}), 1.34(\mathrm{t}, \mathrm{J}=7.2 \mathrm{~Hz}, 3 \mathrm{H}) .{ }^{13} \mathrm{C} \mathrm{NMR}\left(75 \mathrm{MHz}, \mathrm{CDCl}_{3}, \mathrm{ppm}\right): \delta 162.4$, 139.1, 138.5, 130.3, 116.3, 62.7, 47.3, 14.3. 
Entry 6, (E)-methyl 5,5-dibromopenta-2,4-dienoate (20). Following the general procedure for 16, 15 (150 mg, $0.66 \mathrm{mmol})$, methyl acrylate (14) (114 mg, $1.33 \mathrm{mmol})$, and $3(28 \mathrm{mg}, 0.033 \mathrm{mmol})$ in $3.3 \mathrm{~mL} \mathrm{CH}_{2} \mathrm{Cl}_{2}$ gave $70 \mathrm{mg}(39 \%,>20: 1 \mathrm{E} / \mathrm{Z})$ of 20 as a colorless oil. ${ }^{1} \mathrm{H}$ NMR (300 $\left.\mathrm{MHz}, \mathrm{CDCl}_{3}, \mathrm{ppm}\right): \delta 7.16(\mathrm{dd}, \mathrm{J}=16.6,12.8 \mathrm{~Hz}, 1 \mathrm{H}), 6.84$ (d, $12.8 \mathrm{~Hz}, 1 \mathrm{H}), 5.90$ (d, $16.6 \mathrm{~Hz}, 1 \mathrm{H}), 3.61(\mathrm{~s}, 1 \mathrm{H}) .{ }^{13} \mathrm{C} \mathrm{NMR}\left(75 \mathrm{MHz}, \mathrm{CDCl}_{3}, \mathrm{ppm}\right)$ : $\delta 166.9,140.1,135.1,124.1,100.7,52.3$. HRMS (EI) calc. for $\mathrm{C}_{6} \mathrm{H}_{6} \mathrm{Br}_{2} \mathrm{O}_{2}: 269.8735$, found 269.8725 .

Entry 7, (E)-1-(4,4-dibromobuta-1,3-dienyl)benzene (21). Following the general procedure for 16, 15 (150 mg, $0.66 \mathrm{mmol})$, styrene (11) (138 mg, $1.33 \mathrm{mmol})$, and $\mathbf{3}$ (28 $\mathrm{mg}, 0.033 \mathrm{mmol})$ in $3.3 \mathrm{~mL} \mathrm{CH} \mathrm{Cl}_{2}$ gave $115 \mathrm{mg}(60 \%,>20: 1 \mathrm{E} / \mathrm{Z})$ of 21 as a colorless oil. ${ }^{1} \mathrm{H}$ NMR (300 MHz, $\mathrm{CDCl}_{3}$, ppm): $\delta$ 7.44-7.47 (m, 2H), 7.25-7.38 (m, 3H), 7.10 (d, J $=8.9 \mathrm{~Hz}, 1 \mathrm{H}), 6.68-6.84(\mathrm{~m}, 2 \mathrm{H}) .{ }^{13} \mathrm{C} \mathrm{NMR}\left(75 \mathrm{MHz}, \mathrm{CDCl}_{3}, \mathrm{ppm}\right): \delta 137.0,136.2$, 135.6, 128.7, 128.5, 126.7, 125.2, 91.3. HRMS (EI) calc. for $\mathrm{C}_{10} \mathrm{H}_{8} \mathrm{Br}_{2}$ : 287.8972, found 287.8981 .

Entry 8, (E)-8,8-dibromoocta-5,7-dienyl acetate (22). Following the general procedure for 16, 15 (150 mg, $0.66 \mathrm{mmol})$, 5-hexenyl acetate (5) (189 mg, $1.33 \mathrm{mmol})$, and $\mathbf{3}$ (28 $\mathrm{mg}, 0.033 \mathrm{mmol})$ in $3.3 \mathrm{~mL} \mathrm{CH} \mathrm{Cl}_{2}$ gave $122 \mathrm{mg}(56 \%, 5: 1 \mathrm{E} / \mathrm{Z}$ ratio) of 22 as a colorless oil. ${ }^{1} \mathrm{H}$ NMR (300 MHz, $\left.\mathrm{CDCl}_{3}, \mathrm{ppm}\right): \delta 6.86(\mathrm{~d}, \mathrm{~J}=10.0 \mathrm{~Hz}, 1 \mathrm{H}), 6.05$ (ddt, J = 15.2, 10.0, 2.6 Hz, 1H), $5.85(\mathrm{dt}, \mathrm{J}=15.2,7.0 \mathrm{~Hz}, 1 \mathrm{H}), 4.02(\mathrm{t}, \mathrm{J}=6.5 \mathrm{~Hz}, 2 \mathrm{H}), 2.06-2.15(\mathrm{~m}$, 2H), $2.00(\mathrm{~s}, 3 \mathrm{H}), 1.55-1.65(\mathrm{~m}, 2 \mathrm{H}), 1.39-1.50(\mathrm{~m}, 2 \mathrm{H}) .{ }^{13} \mathrm{C} \mathrm{NMR}\left(75 \mathrm{MHz}, \mathrm{CDCl}_{3}\right.$, 
ppm): $\delta 171.0,138.5,136.8,127.5,88.7,64.1,32.6,28.0,25.0,20.9$. HRMS (EI) calc. for $\mathrm{C}_{10} \mathrm{H}_{14} \mathrm{Br}_{2} \mathrm{O}_{2}: 325.9361$, found 325.9346 .

General Procedure for Cross-Metathesis Reactions Using 1,2-Disubstituted 1,3Butadienes (Table 2). Entry 1, 4,4,5,5-Tetramethyl-2-((1E,3E)-3-methylpenta-1,3dienyl)-1,3,2-dioxaborolane and 4,4,5,5-tetramethyl-2-((1E,3Z)-3-methylpenta-1,3dienyl)-1,3,2-dioxaborolane (30). To a solution of $\mathbf{3}$ (14 mg, $0.016 \mathrm{mmol})$ in $\mathrm{CH}_{2} \mathrm{Cl}_{2}$ (1 $\mathrm{mL}$ ) was added 3-methyl-1,3-pentadiene (28) (27 $\mathrm{mg}, 0.32 \mathrm{mmol}$ ) and vinylboronate $\mathbf{2 3}$ (50 $\mathrm{mg}, 0.32 \mathrm{mmol}$ ). The solution stirred at $40{ }^{\circ} \mathrm{C}$ for $12 \mathrm{~h}$, and the solvent was removed by rotary evaporation. The crude product was purified by flash chromatography (5\% ethyl acetate:hexanes) to give $54 \mathrm{mg}(80 \%,>20: 1 \mathrm{E} / \mathrm{Z})$ of the two isomers of $\mathbf{3 0}$. A small amount (10\%) of the cross product missing the terminal methyl group (25) was identified by a broad singlet at $5.15 \mathrm{ppm}$ in the ${ }^{1} \mathrm{H}$ NMR spectrum (terminal $\mathrm{C}=\mathrm{CH}_{2}$ ) and by HRMS (EI) (calc. for $\mathrm{C}_{11} \mathrm{H}_{19} \mathrm{BO}_{2}: 194.1478$, found 194.1485). ${ }^{1} \mathrm{H} \mathrm{NMR} \mathrm{(300} \mathrm{MHz,} \mathrm{CDCl}_{3}$, ppm): $\delta 7.47$ (d, J = 18.1 Hz, 1H, Z-isomer), 7.03 (d, J = 18.1 Hz, 1H, $E$-isomer), 5.76 (q, $\mathrm{J}=6.8 \mathrm{~Hz}, 1 \mathrm{H}, E$-isomer), $5.62(\mathrm{~m}, 1 \mathrm{H}, \mathrm{Z}$-isomer), $5.55(\mathrm{~d}, \mathrm{~J}=18.1 \mathrm{~Hz}, 1 \mathrm{H}, \mathrm{Z}$-isomer $)$, $5.42(\mathrm{~d}, \mathrm{~J}=18.1 \mathrm{~Hz}, 1 \mathrm{H}, E$-isomer), $1.78(\mathrm{~d}, \mathrm{~J}=11.0 \mathrm{~Hz}, 3 \mathrm{H}), 1.73(\mathrm{~s}, 3 \mathrm{H}), 1.26(\mathrm{~s}, 12 \mathrm{H})$. ${ }^{13} \mathrm{C}$ NMR $\left(75 \mathrm{MHz}, \mathrm{CDCl}_{3}\right.$, ppm) of $E$-isomer: $\delta 154.7,131.9,129.1,83.2,25.0,14.5$, 11.5. HRMS (EI) calc. for $\mathrm{C}_{12} \mathrm{H}_{21} \mathrm{BO}_{2}$ (for both isomers): 208.1635 , found 208.1636 and 208.1627.

Entry 2, $(2 E, 4 E)$-4-methylhexa-2,4-dienyl acetate and $(2 E, 4 Z)$-4-methylhexa-2,4dienyl acetate (31). Following the general procedure for 30, 3-methyl-1,3-pentadiene 
(28) (40 mg, $0.49 \mathrm{mmol}$ ), 1,4-diacetoxy-cis-2-butene (12) (167 mg, $0.97 \mathrm{mmol}$ ), and $\mathbf{3}$ (21 mg, $0.024 \mathrm{mmol})$ in $1.5 \mathrm{~mL} \mathrm{CH}_{2} \mathrm{Cl}_{2}$ gave $62 \mathrm{mg}(82 \%,>20: 1 \mathrm{E} / \mathrm{Z})$ of $\mathbf{3 1}$ as a colorless oil. A small amount (9\%) of the cross product missing the terminal methyl group was identified by a broad singlet at $5.00 \mathrm{ppm}$ in the ${ }^{1} \mathrm{H}$ NMR spectrum (terminal $\mathrm{C}=\mathrm{CH}_{2}$ ) and by HRMS (EI) (calc. for $\mathrm{C}_{8} \mathrm{H}_{12} \mathrm{O}_{2}: 140.0837$, found 140.0841). ${ }^{1} \mathrm{H}$ NMR (300 MHz, $\left.\mathrm{CDCl}_{3}, \mathrm{ppm}\right): \delta 6.71$ (d, J = 15.5 Hz, 1H, Z-isomer), 6.29 (d, J = 15.7 Hz, 1H,

E-isomer), 5.44-5.77 (m, 2H, both $E$ - and Z-isomers), 4.64 (d, J = 7.1 Hz, 2H, Z-isomer), 4.59 (d, J = 7.4 Hz, 2H, E-isomer), 2.06 (s, 3H, Z-isomer), 2.05 (s, 3H, E-isomer), 1.81 (d, $\mathrm{J}=15.9 \mathrm{~Hz}, 3 \mathrm{H}$, both isomers), 1.72 (s, $3 \mathrm{H}, E$-isomer), 1.70 (s, $3 \mathrm{H}, \mathrm{Z}$-isomer). ${ }^{13} \mathrm{C}$ NMR (75 MHz, $\left.\mathrm{CDCl}_{3}, \mathrm{ppm}\right)$ of $E$-isomer: $\delta 171.1,139.8,133.8,128.8,119.5,65.7$, 21.2, 14.1, 12.1. HRMS (EI) calc. for $\mathrm{C}_{9} \mathrm{H}_{14} \mathrm{O}_{2}$ (for both isomers): 154.0994, found 154.0987 and 154.0994.

Entry 3, (3E,5E)-5-methylhepta-3,5-dien-2-one and (3E,5Z)-5-methylhepta-3,5-dien2-one (32). Following the general procedure for 30, 3-methyl-1,3-pentadiene (28) (40 $\mathrm{mg}, 0.49 \mathrm{mmol}$ ), methylvinylketone (26) (34 mg, $0.49 \mathrm{mmol}$ ), and $\mathbf{3}$ (21 mg, 0.024 mmol) in $1.5 \mathrm{~mL} \mathrm{CH}_{2} \mathrm{Cl}_{2}$ gave $42 \mathrm{mg}(70 \%,>20: 1 \mathrm{E} / \mathrm{Z})$ of $\mathbf{3 2}$ as a colorless oil. A small amount $(7 \%)$ of the cross product missing the terminal methyl group was identified by a broad singlet at $5.40 \mathrm{ppm}$ in the ${ }^{1} \mathrm{H}$ NMR spectrum (terminal $\mathrm{C}=\mathrm{CH}_{2}$ ) and by HRMS (EI) (calc. for $\mathrm{C}_{7} \mathrm{H}_{10} \mathrm{O}$ : 110.0732 , found 110.0727). ${ }^{1} \mathrm{H}$ NMR (300 MHz, $\mathrm{CDCl}_{3}, \mathrm{ppm}$ ): $\delta 7.26$ (d, J = 15.7 Hz, 1H, Z-isomer), 7.14 (d, J = $15.9 \mathrm{~Hz}, 1 \mathrm{H}, E$-isomer), 6.15 (d, J = $15.9 \mathrm{~Hz}$, 1H, Z-isomer), 6.05 (d, J = 15.9 Hz, 1H, E-isomer), 6.01 (q, J = 7.1 Hz, 1H, E-isomer), 5.88 (q, J = 7.1 Hz, 1H, Z-isomer), 2.32 (s, 3H, Z-isomer), 2.26 (s, 3H, E-isomer), 1.87- 
1.90 (m, 3H, Z-isomer), 1.83-1.87 (m, 3H, Z-isomer), 1.81 (d, J = 7.1 Hz, 3H, E-isomer), 1.76 (s, $3 \mathrm{H}, E$-isomer). ${ }^{13} \mathrm{C}$ NMR (75 MHz, $\mathrm{CDCl}_{3}$, ppm) of $E$-isomer: $\delta 199.2,148.8$, 137.7, 134.3, 125.1, 31.8, 27.5, 22.9. HRMS (EI) calc. for $\mathrm{C}_{8} \mathrm{H}_{12} \mathrm{O}$ (for both isomers): 124.0888, found 124.0882 and 124.0886 .

Entry 4, (5E,7E)-7-propylundeca-5,7-dienyl acetate (33). Following the general procedure for 30, diene $\mathbf{2 9}$ (40 mg, $0.29 \mathrm{mmol}$ ), 5-hexenyl acetate (5) (165 mg, 1.2 mmol), and 3 (12 mg, $0.014 \mathrm{mmol})$ in $1.2 \mathrm{~mL} \mathrm{CH}_{2} \mathrm{Cl}_{2}$ gave $56 \mathrm{mg}(77 \%,>20: 1 \mathrm{E} / \mathrm{Z})$ of 33 as a colorless oil. The product was not separated from unreacted 5 (1.0:0.32 33/5). A small amount (12\%) of the cross product missing the terminal methyl group was identified by 2 broad singlets at 5.84 and $5.88 \mathrm{ppm}$ in the ${ }^{1} \mathrm{H}$ NMR spectrum (terminal $\left.\mathrm{C}=\mathrm{CH}_{2}\right) .{ }^{1} \mathrm{H} \mathrm{NMR}\left(300 \mathrm{MHz}, \mathrm{CDCl}_{3}, \mathrm{ppm}\right): \delta 6.31(\mathrm{~d}, \mathrm{~J}=15.7 \mathrm{~Hz}, 1 \mathrm{H}), 5.64(\mathrm{dt}, \mathrm{J}=$ 15.7, $6.9 \mathrm{~Hz}, 1 \mathrm{H}), 5.24(\mathrm{t}, \mathrm{J}=7.1 \mathrm{~Hz}, 1 \mathrm{H}), 4.06(\mathrm{t}, \mathrm{J}=6.6 \mathrm{~Hz}, 2 \mathrm{H}), 2.06-2.19(\mathrm{~m}, 6 \mathrm{H})$, $2.04(\mathrm{~s}, 3 \mathrm{H}), 1.58-1.70(\mathrm{~m}, 2 \mathrm{H}), 1.38-1.52(\mathrm{~m}, 6 \mathrm{H}), 0.89(\mathrm{q}, \mathrm{J}=6.0 \mathrm{~Hz}, 6 \mathrm{H}) .{ }^{13} \mathrm{C}$ NMR (75 MHz, $\left.\mathrm{CDCl}_{3}, \mathrm{ppm}\right): \delta 171.4,136.1,129.2,128.7,127.0,64.6,36.5,33.1,29.6,28.3$, 26.1, 23.3, 22.2, 21.2, 14.2, 14.0. HRMS (EI) calc. for $\mathrm{C}_{16} \mathrm{H}_{28} \mathrm{O}_{2}: 252.2089$, found 252.2094 .

Entry 5, (2E,4E)-4-propylocta-2,4-dienyl benzoate (34). Following the general procedure for 30, diene $\mathbf{2 9}$ (40 mg, $0.29 \mathrm{mmol}$ ), 1,4-dibenzoyl-2-butene (27) (171 mg, $0.58 \mathrm{mmol})$, and $\mathbf{3}(12 \mathrm{mg}, 0.014 \mathrm{mmol})$ in $1.4 \mathrm{~mL} \mathrm{CH}_{2} \mathrm{Cl}_{2}$ gave $62 \mathrm{mg}(79 \%,>20: 1 \mathrm{E} / \mathrm{Z})$ of $\mathbf{3 4}$ as a colorless oil. The product was not separated from allyl benzoate formed in the reaction (1.0:0.25 34/allyl benzoate). ${ }^{1} \mathrm{H}$ NMR (300 MHz, $\left.\mathrm{CDCl}_{3}, \mathrm{ppm}\right): \delta 8.05-8.10$ (m, 
2H), 7.52-7.59 (m, 1H), 7.41-7.48 (m, 2H), $6.70(\mathrm{dd}, \mathrm{J}=15.8,1.1 \mathrm{~Hz}, 1 \mathrm{H}), 5.88(\mathrm{dt}, \mathrm{J}=$ 15.7, $6.3 \mathrm{~Hz}, 1 \mathrm{H}), 5.43(\mathrm{t}, \mathrm{J}=7.4 \mathrm{~Hz}, 1 \mathrm{H}), 4.91(\mathrm{dd}, \mathrm{J}=6.6,1.1 \mathrm{~Hz}, 2 \mathrm{H}), 2.12-2.21(\mathrm{~m}$, 4H), 1.35-1.55 (m, 4H), $0.92(\mathrm{t}, \mathrm{J}=7.4 \mathrm{~Hz}, 3 \mathrm{H}), 0.91(\mathrm{t}, \mathrm{J}=7.4 \mathrm{~Hz}, 3 \mathrm{H}) .{ }^{13} \mathrm{C}$ NMR $(75$ $\left.\mathrm{MHz}, \mathrm{CDCl}_{3}, \mathrm{ppm}\right): \delta 166.6,135.4,133.1,132.1,131.2,129.8,128.8,128.5,122.2,66.5$, 36.2, 29.7, 23.3, 22.0, 14.2, 14.0. HRMS (EI) calc. for $\mathrm{C}_{18} \mathrm{H}_{24} \mathrm{O}_{2}$ : 272.1776, found 272.1777 .

\section{General Procedure for Cross-Metathesis Reactions Using 2-Substituted 1,3-}

Butadienes (Table 3). Entry 1, (E)-4-methylenedec-2-enyl acetate (36). To a solution of $\mathbf{3}(12 \mathrm{mg}, 0.014 \mathrm{mmol})$ in benzene $(1.5 \mathrm{~mL})$ was added 1,4-diacetoxy-cis-2-butene (12) (100 mg, $0.58 \mathrm{mmol})$ and diene 35 (40 $\mathrm{mg}, 0.29 \mathrm{mmol})$. The solution stirred at 60 ${ }^{\circ} \mathrm{C}$ for $12 \mathrm{~h}$, and the solvent was removed by rotary evaporation. The crude product was purified by flash chromatography (5\% ethyl acetate:hexanes) to give $44 \mathrm{mg}(72 \%,>20: 1$ E/Z) of a colorless oil. ${ }^{1} \mathrm{H}$ NMR (300 MHz, $\left.\mathrm{CDCl}_{3}, \mathrm{ppm}\right): \delta 6.23(\mathrm{~d}, \mathrm{~J}=16.0 \mathrm{~Hz}, 1 \mathrm{H})$, $5.70(\mathrm{dt}, \mathrm{J}=15.7,6.6 \mathrm{~Hz}, 1 \mathrm{H}), 4.96(\mathrm{br} \mathrm{s}, 1 \mathrm{H}), 4.94(\mathrm{br} \mathrm{s}, 1 \mathrm{H}), 4.55(\mathrm{dd}, \mathrm{J}=6.3,1.1 \mathrm{~Hz}$, 2H), $2.11(\mathrm{t}, \mathrm{J}=7.0 \mathrm{~Hz}, 2 \mathrm{H}), 2.01(\mathrm{~s}, 3 \mathrm{H}), 1.36-1.43(\mathrm{~m}, 2 \mathrm{H}), 1.18-1.27$ (m, 6H), 0.80$0.84(\mathrm{~m}, 3 \mathrm{H}) .{ }^{13} \mathrm{C} \mathrm{NMR}\left(75 \mathrm{MHz}, \mathrm{CDCl}_{3}, \mathrm{ppm}\right): \delta 171.0,145.6,136.8,122.4,116.7$, 65.4, 32.0, 31.9, 29.4, 28.2, 22.8, 21.2, 14.3. HRMS (EI) calc. for $\mathrm{C}_{13} \mathrm{H}_{22} \mathrm{O}_{2}: 210.1620$, found 210.1616 .

Entry 2, $(E)$-4-methylenedec-2-enyl benzoate (39). Following the general procedure for 36, diene 35 (40 mg, $0.29 \mathrm{mmol}$ ), 1,4-dibenzoyl-2-butene (27) (171 mg, $0.58 \mathrm{mmol}$ ), and $3(12 \mathrm{mg}, 0.014 \mathrm{mmol})$ in $1.4 \mathrm{~mL}$ benzene gave $57 \mathrm{mg}(73 \%,>20: 1 \mathrm{E} / \mathrm{Z})$ of $\mathbf{3 9}$ as a 
colorless oil. The product was not separated from allyl benzoate formed in the reaction (1.0:0.46 39/allyl benzoate). ${ }^{1} \mathrm{H}$ NMR (300 MHz, $\left.\mathrm{CDCl}_{3}, \mathrm{ppm}\right): \delta 8.05-8.09$ (m, 2H), 7.53-7.60 (m, 1H), $7.44(\mathrm{t}, \mathrm{J}=7.4 \mathrm{~Hz}, 2 \mathrm{H}), 6.40(\mathrm{~d}, \mathrm{~J}=15.7 \mathrm{~Hz}, 1 \mathrm{H}), 5.90(\mathrm{dt}, \mathrm{J}=15.9$, $6.3 \mathrm{~Hz}, 1 \mathrm{H}), 5.06$ (br s, 1H), $5.03(\mathrm{br} \mathrm{s}, 1 \mathrm{H}), 4.88(\mathrm{dd}, \mathrm{J}=6.3,1.1 \mathrm{~Hz}, 2 \mathrm{H}), 2.22(\mathrm{t}, \mathrm{J}=$ $6.9 \mathrm{~Hz}, 2 \mathrm{H}), 1.45-1.58(\mathrm{~m}, 2 \mathrm{H}), 1.27-1.36(\mathrm{~m}, 6 \mathrm{H}), 0.86-0.91(\mathrm{~m}, 3 \mathrm{H}) .{ }^{13} \mathrm{C} \mathrm{NMR}(75$ $\left.\mathrm{MHz}, \mathrm{CDCl}_{3}, \mathrm{ppm}\right): \delta 166.6,145.6,136.8,133.2,133.1,129.8,128.5,122.6,116.7,65.8$, 32.1, 31.9, 29.4, 28.2, 22.8, 14.2. HRMS (EI) calc. for $\mathrm{C}_{18} \mathrm{H}_{24} \mathrm{O}_{2}: 272.1776$, found 272.1778 .

\section{Entry 3, (E)-4,4,5,5-tetramethyl-2-(3-methylenenon-1-enyl)-1,3,2-dioxaborolane} (40). Following a slight modification of the general procedure for $\mathbf{3 6}$, vinyl boronate $\mathbf{2 3}$ (89 mg, $0.58 \mathrm{mmol}$ ), diene 35, (40 mg, $0.29 \mathrm{mmol})$, and $\mathbf{3}$ (25 mg, $0.029 \mathrm{mmol})$ in 1.5 $\mathrm{mL}$ benzene for $2 \mathrm{~h}$ at $60{ }^{\circ} \mathrm{C}$ (followed by the same work-up) gave $56 \mathrm{mg}(73 \%,>20: 1$ E/Z) of a yellow oil. ${ }^{1} \mathrm{H}$ NMR (300 MHz, $\left.\mathrm{CDCl}_{3}, \mathrm{ppm}\right): \delta 7.03(\mathrm{~d}, \mathrm{~J}=18.4 \mathrm{~Hz}, 1 \mathrm{H}), 5.59$ (d, J = 18.4 Hz, 1H), 5.16 (br s, 1H), 5.13 (br s, 1H), $2.20(\mathrm{t}, \mathrm{J}=7.4 \mathrm{~Hz}, 2 \mathrm{H}), 1.41-1.48$ $(\mathrm{m}, 2 \mathrm{H}), 1.20-1.34(\mathrm{~m}, 6 \mathrm{H}), 1.27(\mathrm{~s}, 12 \mathrm{H}), 0.85-0.89(\mathrm{~m}, 3 \mathrm{H}) .{ }^{13} \mathrm{C}$ NMR $(75 \mathrm{MHz}$, $\left.\mathrm{CDCl}_{3}, \mathrm{ppm}\right): \delta 152.0,147.6,119.3,83.4,31.9,31.5,29.5,28.5,25.0,22.9,14.3$. HRMS (EI) calc. for $\mathrm{C}_{16} \mathrm{H}_{29} \mathrm{BO}_{2}$ : 264.2261, found 264.2251.

Entry 4, (E)-6-(tert-butyldimethylsilyloxy)-4-methylenehex-2-enyl benzoate (41).

Following the general procedure for 36, diene $\mathbf{3 7}$ (40 mg, 0.19 mmol), 1,4-dibenzoyl-2butene (27) (112 $\mathrm{mg}, 0.38 \mathrm{mmol})$, and $\mathbf{3}(8 \mathrm{mg}, 0.009 \mathrm{mmol})$ in $1 \mathrm{~mL}$ benzene gave 46 $\mathrm{mg}(70 \%,>20: 1 \mathrm{E} / \mathrm{Z})$ of $\mathbf{4 1}$ as a colorless oil. ${ }^{1} \mathrm{H} \mathrm{NMR}\left(300 \mathrm{MHz}, \mathrm{CDCl}_{3}, \mathrm{ppm}\right): \delta 8.05-$ 
$8.10(\mathrm{~m}, 2 \mathrm{H}), 7.53-7.59(\mathrm{~m}, 1 \mathrm{H}), 7.41-7.47(\mathrm{~m}, 2 \mathrm{H}), 6.39(\mathrm{~d}, \mathrm{~J}=15.9 \mathrm{~Hz}, 1 \mathrm{H}), 5.92(\mathrm{dt}, \mathrm{J}$ $=15.9,6.3 \mathrm{~Hz}, 1 \mathrm{H}), 5.12(\mathrm{br} \mathrm{s}, 1 \mathrm{H}), 5.07(\mathrm{br} \mathrm{s}, 1 \mathrm{H}), 4.88(\mathrm{~d}, \mathrm{~J}=6.3 \mathrm{~Hz}, 2 \mathrm{H}), 3.75(\mathrm{t}, \mathrm{J}=$ $7.1 \mathrm{~Hz}, 2 \mathrm{H}), 2.47(\mathrm{t}, \mathrm{J}=7.1 \mathrm{~Hz}, 2 \mathrm{H}), 0.89(\mathrm{~s}, 9 \mathrm{H}), 0.05(\mathrm{~s}, 6 \mathrm{H}) .{ }^{13} \mathrm{C} \mathrm{NMR}(75 \mathrm{MHz}$, $\left.\mathrm{CDCl}_{3}, \mathrm{ppm}\right): \delta 166.5,142.3,136.6,133.2,130.4,129.8,128.5,122.9,118.6,65.7,62.4$, 35.7, 26.1, 18.5, -5.1. HRMS (EI) calc. for $\mathrm{C}_{20} \mathrm{H}_{31} \mathrm{O}_{3} \mathrm{Si}[\mathrm{M}+\mathrm{H}]: 347.2043$, found 347.2047.

\section{Entry 5, (E)-9-(tert-butyldimethylsilyloxy)-7-methylenenon-5-enyl acetate (42).}

Following the procedure for 36, diene $\mathbf{3 7}$ (40 mg, $0.19 \mathrm{mmol}$ ), 5-hexenyl acetate (5) (107 $\mathrm{mg}, 0.75 \mathrm{mmol})$, and $\mathbf{3}(8 \mathrm{mg}, 0.009 \mathrm{mmol})$ in $1 \mathrm{~mL}$ benzene gave $46 \mathrm{mg}(75 \%,>20: 1$ E/Z) of 42 as a colorless oil. ${ }^{1} \mathrm{H}$ NMR $\left(300 \mathrm{MHz}, \mathrm{CDCl}_{3}, \mathrm{ppm}\right): \delta 6.05(\mathrm{~d}, \mathrm{~J}=15.7 \mathrm{~Hz}$, 1H), $5.69(\mathrm{dt}, \mathrm{J}=15.9,6.9 \mathrm{~Hz}, 1 \mathrm{H}), 4.94(\mathrm{br} \mathrm{s}, 1 \mathrm{H}), 4.88(\mathrm{br} \mathrm{s}, 1 \mathrm{H}), 4.06(\mathrm{t}, \mathrm{J}=6.6 \mathrm{~Hz}$, 2H), $3.71(\mathrm{t}, \mathrm{J}=7.1 \mathrm{~Hz}, 2 \mathrm{H}), 2.43(\mathrm{t}, \mathrm{J}=6.9 \mathrm{~Hz}, 2 \mathrm{H}), 2.13(\mathrm{q}, \mathrm{J}=6.9 \mathrm{~Hz}, 2 \mathrm{H}), 2.04(\mathrm{~s}$, $3 \mathrm{H}), 1.58-1.68(\mathrm{~m}, 2 \mathrm{H}), 1.41-1.51(\mathrm{~m}, 2 \mathrm{H}), 0.89(\mathrm{~s}, 9 \mathrm{H}), 0.04(\mathrm{~s}, 6 \mathrm{H}) .{ }^{13} \mathrm{C} \mathrm{NMR}(75$ $\left.\mathrm{MHz}, \mathrm{CDCl}_{3}, \mathrm{ppm}\right): \delta 171.4,143.0,132.7,129.8,115.3,64.6,62.8,36.0,32.5,28.3$, 26.2, 25.9, 21.2, 18.6, -5.1. HRMS (EI) calc. for $\mathrm{C}_{18} \mathrm{H}_{35} \mathrm{O}_{3} \mathrm{Si}[\mathrm{M}+\mathrm{H}]$ : 327.2356, found 327.2366 .

\section{Entry 6, (E)-tert-butyldimethyl(3-methylene-5-(4,4,5,5-tetramethyl-1,3,2-}

dioxaborolan-2-yl)pent-4-enyloxy)silane (43). Following a slight modification of the general procedure for 36, diene $\mathbf{3 7}$ (40 mg, $0.19 \mathrm{mmol}$ ), vinyl boronate $\mathbf{2 3}$ (59 mg, 0.38 $\mathrm{mmol}$ ), and $\mathbf{3}(16 \mathrm{mg}, 0.019 \mathrm{mmol})$ in $1 \mathrm{~mL}$ benzene for $2 \mathrm{~h}$ at $60^{\circ} \mathrm{C}$ (followed by the same work-up) gave $44 \mathrm{mg}(69 \%,>20: 1 \mathrm{E} / \mathrm{Z})$ of $\mathbf{4 3}$ as a yellow oil. ${ }^{1} \mathrm{H}$ NMR $(300 \mathrm{MHz}$, 
$\left.\mathrm{CDCl}_{3}, \mathrm{ppm}\right): \delta 7.03(\mathrm{~d}, \mathrm{~J}=18.4 \mathrm{~Hz}, 1 \mathrm{H}), 5.60(\mathrm{~d}, \mathrm{~J}=18.4 \mathrm{~Hz}, 1 \mathrm{H}), 5.23(\mathrm{br} \mathrm{s}, 1 \mathrm{H}), 5.18$ (br s, 1H), $3.70(\mathrm{t}, \mathrm{J}=7.1 \mathrm{~Hz}, 2 \mathrm{H}), 2.46(\mathrm{dt}, \mathrm{J}=7.1,1.1 \mathrm{~Hz}, 2 \mathrm{H}), 1.27(\mathrm{~s}, 12 \mathrm{H}), 0.87$ (s, 9H), 0.02 (s, 6H). $\left.{ }^{13} \mathrm{C} \mathrm{NMR} \mathrm{(75} \mathrm{MHz,} \mathrm{CDCl}_{3}, \mathrm{ppm}\right): \delta 151.8,144.1,121.0,83.4,62.4$, 35.0, 26.1, 25.0, 18.5, -5.1. HRMS (EI) calc. for $\mathrm{C}_{18} \mathrm{H}_{35} \mathrm{BO}_{3} \mathrm{Si}$ : 338.2449, found 338.2455.

Entry 7, (E)-tert-butyldimethyl(2-methylene-4-(4,4,5,5-tetramethyl-1,3,2dioxaborolan-2-yl)but-3-enyloxy)silane (44). Following a slight modification of the general procedure for $\mathbf{3 6}$, diene $\mathbf{3 8}$ ( $40 \mathrm{mg}, 0.20 \mathrm{mmol}$ ), vinyl boronate $\mathbf{2 3}$ (62 mg, 0.40 $\mathrm{mmol}$ ), and $\mathbf{3}(17 \mathrm{mg}, 0.020)$ in $1 \mathrm{~mL}$ benzene for $2 \mathrm{~h}$ at $60{ }^{\circ} \mathrm{C}$ (followed by the same work-up) gave $37 \mathrm{mg}$ (approximately 73\% pure; 40\% yield based on impurities and unreacted, inseparable boronate $\mathbf{2 3},>20: 1 \mathrm{E} / \mathrm{Z}$ ) of impure $\mathbf{4 4}$ as a yellow oil. Peaks given in spectral data are only those corresponding to $\left.44 .{ }^{1} \mathrm{H} \mathrm{NMR} \mathrm{(300} \mathrm{MHz,} \mathrm{CDCl}_{3}, \mathrm{ppm}\right): \delta$ $7.06(\mathrm{~d}, \mathrm{~J}=18.9 \mathrm{~Hz}, 1 \mathrm{H}), 5.49(\mathrm{q}, \mathrm{J}=1.9 \mathrm{~Hz}, 1 \mathrm{H}), 5.48(\mathrm{~d}, \mathrm{~J}=18.7 \mathrm{~Hz}, 1 \mathrm{H}), 5.28(\mathrm{br} \mathrm{s}$, 1H), $4.36(\mathrm{t}, \mathrm{J}=1.6 \mathrm{~Hz}, 2 \mathrm{H}), 1.28(\mathrm{~s}, 12 \mathrm{H}), 0.92(\mathrm{~s}, 9 \mathrm{H}), 0.07$ (s, 6H).

\section{Entry 8, (E)-4-((tert-butyldimethylsilyloxy)methyl)penta-2,4-dienyl benzoate (45).}

Following the general procedure for 36, diene $\mathbf{3 8}$ ( $40 \mathrm{mg}, 0.20 \mathrm{mmol}$ ), 1,4-dibenzoyl-2butene (27) (119 mg, 0.40), and $\mathbf{3}$ (9 mg, $0.01 \mathrm{mmol}$ ) in $1 \mathrm{~mL}$ benzene gave $42 \mathrm{mg}$ (63\%, $>20: 1 \mathrm{E} / \mathrm{Z}$ ) of $\mathbf{4 5}$ as a pale yellow oil. Compound $\mathbf{4 5}$ was not separated from allyl benzoate formed in the reaction (1.0:0.55 45:allyl benzoate). ${ }^{1} \mathrm{H}$ NMR $(300 \mathrm{MHz}$, $\left.\mathrm{CDCl}_{3}, \mathrm{ppm}\right): \delta$ 8.03-8.09 (m, 2H), 7.53-7.59 (m, 1H), $7.44(\mathrm{t}, \mathrm{J}=8.0 \mathrm{~Hz}, 2 \mathrm{H}), 6.40(\mathrm{~d}, \mathrm{~J}$ $=15.9 \mathrm{~Hz}, 1 \mathrm{H}), 5.87(\mathrm{dt}, \mathrm{J}=15.9,6.3 \mathrm{~Hz}, 1 \mathrm{H}), 5.37(\mathrm{br} \mathrm{s}, 1 \mathrm{H}), 5.17(\mathrm{br} \mathrm{s}, 1 \mathrm{H}), 4.86(\mathrm{~d}, \mathrm{~J}$ 
$=6.3 \mathrm{~Hz}, 2 \mathrm{H}), 4.35(\mathrm{~s}, 2 \mathrm{H}), 0.92(\mathrm{~s}, 9 \mathrm{H}), 0.09(\mathrm{~s}, 6 \mathrm{H}) .{ }^{13} \mathrm{C} \mathrm{NMR}\left(75 \mathrm{MHz}, \mathrm{CDCl}_{3}, \mathrm{ppm}\right)$ :

$\delta$ 166.5, 144.0, 134.0, 133.2, 129.8, 128.5, 122.7, 118.4, 116.1, 65.8, 62.9, 26.1, 18.5,

-5.2. HRMS (EI) calc. for $\mathrm{C}_{19} \mathrm{H}_{29} \mathrm{O}_{3} \mathrm{Si}[\mathrm{M}+\mathrm{H}]$ : 333.1886, found 333.1888 .

(E)-1-(3-methylpenta-1,3-dienyl)benzene (46). To a solution of 3 (14 mg, $0.016 \mathrm{mmol})$ in benzene ( $1.5 \mathrm{~mL}$ ) was added vinylboronate $\mathbf{2 3}(50 \mathrm{mg}, 0.32 \mathrm{mmol}$ ) and diene $\mathbf{2 8}$ (26 $\mathrm{mg}, 0.32 \mathrm{mmol}$ ), and the solution stirred at $40{ }^{\circ} \mathrm{C}$ for $2.5 \mathrm{~h}$. The reaction solution was cooled to rt, and $\mathrm{Pd}\left(\mathrm{PPh}_{3}\right)_{4}(11 \mathrm{mg}, 0.0097 \mathrm{mmol})$, bromobenzene $(50 \mathrm{mg}, 0.32 \mathrm{mmol})$, and $\mathrm{NaOEt}(2 \mathrm{M}$ in EtOH, $0.46 \mathrm{~mL}, 0.91 \mathrm{mmol})$ were added. The solution stirred at 80 ${ }^{\circ} \mathrm{C}$ for $5 \mathrm{~h}$. The reaction mixture was purified by flash chromatography (100\% hexanes) to give $23 \mathrm{mg}(45 \%)$ of $\mathbf{4 6}$ as a colorless oil. A small amount (13\%) of the cross product missing the terminal methyl group was identified by two broad singlets at $5.09 \mathrm{ppm}$ and $5.14 \mathrm{ppm}$ in the ${ }^{1} \mathrm{H}$ NMR spectrum (terminal $\mathrm{C}=\mathrm{CH}_{2}$ ). Characterization data matched that in the literature. ${ }^{\text {vii }}{ }^{1} \mathrm{H}$ NMR (300 MHz, $\left.\mathrm{CDCl}_{3}, \mathrm{ppm}\right): \delta$ 7.41-7.48 (m, 2H, both $E$ and $Z$-isomers), 7.28-7.36 (m, 2H, both $E$ - and $Z$-isomers), 7.18-7.26 (m, $1 \mathrm{H}$, both $E$ - and Z-isomers), 6.90 (d, J = 16.2 Hz, 1H, Z-isomer), 6.83 (d, J = 16.2 Hz, 1H, E-isomer), 6.57 (d, J = 15.9 Hz, 1H, Z-isomer), $6.46(\mathrm{~d}, \mathrm{~J}=15.9 \mathrm{~Hz}, 1 \mathrm{H}, E$-isomer), $5.73(\mathrm{q}, \mathrm{J}=7.1 \mathrm{~Hz}$, 1H, $E$-isomer), 5.56 (q, J = 7.1 Hz, 1H, Z-isomer), 1.95 (m, 3H, Z-isomer), 1.88 (t, J = 1.1 $\mathrm{Hz}, 3 \mathrm{H}, E$-isomer), 1.82 (d, J = 7.1 Hz, 3H, E-isomer); terminal methyl resonance of $Z$ isomer overlaps with those of the major isomer.

\footnotetext{
${ }^{\mathrm{i}}$ Braun, N.A.; Burkle, U.; Feth, M.P.; Klein, I.; Spitzner, D. Eur. J. Org. Chem. 1998, 8, 1569-1576.

${ }^{\text {ii }}$ Wrobel, J.E.; Ganem, B. J. Org. Chem. 1983, 48, 3761-3764.
} 
iii Brown, P. A.; Bonnert, R. V.; Jenkins, P. R.; Lawrence, N. J.; Selim, M. R. J. Chem. Soc. Perkin. Trans. 1 1991, 8, 1893-1900.

${ }^{\text {iv }}$ Kamiya, N.; Chikami, Y.; Ishii, Y. Synlett 1990, 675-676.

${ }^{v}$ Basil, L. F.; Nakano, H.; Frutos, R.; Kopach, M.; Meyers, A. I. Synthesis 2002, 14, 2064-2074.

${ }^{v i}$ Lai, M.-T.; Li, D.; Oh, E.; Liu, H.-W. J. Am. Chem. Soc. 1993, 115, 1619-1628.

${ }^{v i i}$ Littke, A. F.; Fu, G. C. J. Am. Chem. Soc. 2001, 123, 6989-7000. 